Bull. Austral. Math. Soc.

$20 \mathrm{E} 45,20 \mathrm{DXX}$

VOL. $69(2004) \quad[317-325]$

\title{
PRIME POWERS AS CONJUGACY CLASS LENGTHS OF $\pi$-ELEMENTS
}

\author{
Antonio Beltrán and María José Felipe
}

\begin{abstract}
Let $G$ be a finite group and $\pi$ an arbitrary set of primes. We investigate the structure of $G$ when the lengths of the conjugacy classes of its $\pi$-elements are prime powers. Under this condition, we show that such lengths are either powers of just one prime or exactly $\left\{1, q^{a}, r^{b}\right\}$, with $q$ and $r$ two distinct primes lying in $\pi$ and $a, b>0$. In the first case, we obtain certain properties of the normal structure of $G$, and in the second one, we provide a characterisation of the structure of $G$.
\end{abstract}

\section{INTRODUCTION}

If $G$ is a finite group, there are many theorems showing that the conjugacy class lengths of $G$ strongly control the structure of $G$. Moreover, several results show that imposing arithmetical conditions on the conjugacy class lengths of certain elements of $G$, for instance when such lengths are prime powers, also reflect on the structure of $G$. In [1], Baer proved that if every element of prime power order of $G$ has a conjugacy class of prime power size, then $G$ is a direct product of factors of coprime orders, each of which is either a p-group or a group with Abelian Sylow subgroups whose order is divisible by just two primes. Recently, Camina and Camina ([7]) restricted the above hypotheses, studying the structure of those groups whose $q$-elements, for some fixed prime $q$, have conjugacy classes of prime power size ( $q$-Baer groups). On the other hand, several authors obtained a complete characterisation of those finite groups whose conjugacy class lengths are prime powers ([8, Theorem 2 and Corollary 2.2], or [6, Theorem 3]). The authors have studied the structure of $p$-solvable groups whose $p^{\prime}$-elements have conjugacy classes of prime power size ([4, Theorem D]).

Let $\pi$ be an arbitrary set of primes and denote by $G_{\pi}$ the set of $\pi$-elements of a group $G$ and by $\operatorname{Con}\left(G_{\pi}\right)$ the set of conjugacy classes in $G_{\pi}$. In this paper, we extend some of the above mentioned results, describing the structure of $G$ when every class in $\operatorname{Con}\left(G_{\pi}\right)$ has prime power size. We recall that a group $G$ is said to be quasi-Frobenius (following [5]) when $G / \mathrm{Z}(G)$ is a Frobenius group. Then we shall refer to the kernel and complement of $G$ as the inverse image in $G$ of the kernel and a complement of $G / Z(G)$. We state our main result.

Received 14th October, 2003

Copyright Clearance Centre, Inc. Serial-fee code: 0004-9727/04 \$A2.00+0.00. 
THEOREM A. Let $G$ be a finite group. Suppose that $|C|$ is a prime power for any $C \in \operatorname{Con}\left(G_{\pi}\right)$. Then one of the two following possibilities occurs.

(a) $|C|$ is a $q$-power for some fixed prime $q$. Moreover,

(1) $q \notin \pi$ if and only if $G$ has an Abelian Hall $\pi$-subgroup $H$. In this case $H \mathbf{O}_{q}(G) \unlhd G$.

(2) $q \in \pi$ if and only if $G=H \times K$ where $K$ is a $\pi$-complement and $H$ is a nilpotent Hall $\pi$-subgroup having all Abelian Sylow subgroups except for the prime $q$.

(b) The lengths of the conjugacy classes in $\operatorname{Con}\left(G_{\pi}\right)$ are powers of exactly two different primes, say $q$ and $r$. This happens if and only if $q, r \in \pi$ and $G=H \times K$, where $K$ is a $\pi$-complement of $G$ and $H$ is a Hall $\pi$-subgroup which is a quasi-Frobenius group with Abelian kernel and complement of orders $q^{a}$ and $r^{b}$ for some $a, b>0$. In particular, the set of conjugacy class lengths in $\operatorname{Con}\left(G_{\pi}\right)$ is exactly $\left\{1, q^{a}, r^{b}\right\}$.

Furthermore, in all cases $G$ has $\pi$-length 1 and $G / O_{\pi^{\prime}}(G)$ is solvable.

As a result of Theorem $\mathrm{A}$, when $\pi=\{p\}$ we provide another proof of $[7$, Theorem $\mathrm{A}(\mathrm{b})]$, which is the following.

Corollary B. Let $G$ be a group and $p$ a fixed prime such that all the conjugacy classes of $p$-elements have prime power size. Then there exists only one prime $q$, equal to or distinct from $p$, such that all classes of $p$-elements have q-power size.

We remark that the proof given there depends, among others, on a result of Kazarin ([10]), requiring Modular Representation Theory. However, our proof is simpler since we only make use of Burnside's $p^{a}$-Lemma to prove the fact that groups satisfying the hypotheses of Theorem A are $\pi$-separable.

One of the main tools to show Theorem A will be to define and study a graph $\Gamma_{\pi}(G)$ associated to the set of non-central classes in $\operatorname{Con}\left(G_{\pi}\right)$ of a $\pi$-separable group $G$. This graph generalises the graphs $\Gamma(G)$ and $\Gamma_{p}(G)$, which were defined and studied earlier in $[5,11,2,3]$. We shall obtain some properties of the maximal size classes in $\operatorname{Con}\left(G_{\pi}\right)$, as we did for $\pi=\left\{p^{\prime}\right\}$ in [3], so as to show Theorem $\mathrm{A}(\mathrm{b})$.

In order to prove Theorem A(a) we have also developed certain properties relating to conjugacy class lengths in $\pi$-separable groups. The most remarkable one is the following extension of a Theorem of Wielandt.

THEOREM C. Let $G$ be a $\pi$-separable group. If $x \in G$ with $\left|x^{G}\right|$ a $\pi$-number, then $\left[x^{G}, x^{G}\right] \subseteq \mathbf{O}_{\pi}(G)$. Consequently, $x \in \mathbf{O}_{\pi, \pi^{\prime}}(G)$.

\section{ThE GRAPH $\Gamma_{\pi}(G)$}

LEMMA 1. Let $G$ be a $\pi$-separable group and let $B=b^{G}, C=c^{G} \in \operatorname{Con}\left(G_{\pi}\right)$ such that $(|B|,|C|)=1$. Then

(a) $C_{G}(b) C_{G}(c)=G$. 
(b) $B C=C B$ is a conjugacy class in $\operatorname{Con}\left(G_{\pi}\right)$ and $|B C|$ divides $|B||C|$.

(c) Let $B_{0} \in \operatorname{Con}\left(G_{\pi}\right)$ of maximal length and assume that $\left(\left|B_{0}\right|,|C|\right)=1$. Then $C^{-1} C B_{0}=B_{0}$ and $\left|\left\langle C^{-1} C\right\rangle\right|$ divides $\left|B_{0}\right|$.

Proof: (a) and (b) Mimic the proof of [11, Lemma 1], replacing the set $p^{\prime}$ by $\pi$.

(c) We know by (b) that $C B_{0} \in \operatorname{Con}\left(G_{\pi}\right)$ and by maximality $\left|C B_{0}\right|=\left|B_{0}\right|$. Again by (b), we have that $C^{-1} C B_{0}$ is a class containing $B_{0}$, so $C^{-1} C B_{0}=B_{0}$. Thus, $\left\langle C^{-1} C\right\rangle B_{0}$ $=B_{0}$ and consequently, $B_{0}$ is union of some cosets of the normal subgroup $\left\langle C^{-1} C\right\rangle$. Then $\left|\left\langle C^{-1} C\right\rangle\right|$ divides $\left|B_{0}\right|$.

We are going to use some properties of the following graph, which is a generalisation of the graph $\Gamma(G)$, defined for ordinary conjugacy classes (see [5]) and the graph $\Gamma_{p}(G)$, defined for $p$-regular classes (see [2]). We define $\Gamma_{\pi}(G)$ to be the graph having as vertices the non-central classes in $\operatorname{Con}\left(G_{\pi}\right)$ and two classes, $C$ and $D$, are connected by an edge when there exists a common prime dividing $|C|$ and $|D|$.

THEOREM 2. Suppose that $G$ is $\pi$-separable. Then $\Gamma_{\pi}(G)$ has at most two connected components.

Proof: It is sufficient to rewrite the proof of [2, Theorem 1], replacing $p^{\prime}$ by $\pi$ and using Lemma 1 (b).

In [3, Theorem 2], the authors obtained some properties related to $p$-regular conjugacy classes of maximal size in $p$-solvable groups. The following theorem extends them for conjugacy classes of $\pi$-elements of maximal size in $\pi$-separable groups.

From now on, $\pi(m)$ will denote the set of prime divisors of a positive integer $m$. We shall also write $\pi(X)$ to denote the set of primes dividing $|X|$ for any group $X$ or any $X \in \operatorname{Con}\left(G_{\pi}\right)$.

THEOREM 3. Suppose that $G$ is $\pi$-separable. Let $B_{0} \in \operatorname{Con}\left(G_{\pi}\right)$ of maximal length and write

$$
M=\left\langle D \in \operatorname{Con}\left(G_{\pi}\right) \mid\left(|D|,\left|B_{0}\right|\right)=1\right\rangle .
$$

Then $M$ is an Abelian $\pi$-subgroup of $G$. Furthermore, $Z_{\pi}:=\mathbf{Z}(G)_{\pi} \subseteq M$ and $\pi\left(M / Z_{\pi}\right)$ $\subseteq \pi\left(B_{0}\right)$.

Proof: We define $N=\left\langle D^{-1} D \mid D \in \operatorname{Con}\left(G_{p^{\prime}}\right),\left(|D|,\left|B_{0}\right|\right)=1\right\rangle$. From the definition of $M$ and $N$, it is clear that $N=[M, G]$. On the other hand, if $C \in \operatorname{Con}\left(G_{\pi}\right)$ with $\left(|C|,\left|B_{0}\right|\right)=1$, then $C^{-1} C B_{0}=B_{0}$ by Lemma $1(\mathrm{c})$, and hence, $N B_{0}=B_{0}$. This means that $B_{0}$ is union of cosets of $N$, so $|N|$ divides $\left|B_{0}\right|$ and $\pi(N) \subseteq \pi\left(B_{0}\right)$. Now, for such a class $c^{G}=C$, we have $(|N|,|C|)=1$. Since $\left|N: C_{N}(c)\right|$ divides $(|N|,|C|)$, it follows that $N=C_{N}(c)$, so $N \leqslant \mathbf{Z}(M)$. But $M / N$ is contained in the centre of $G / N$, and this forces $M$ to be nilpotent. As all the generators of $M$ lie in the Hall $\pi$-subgroup of $M$, we conclude that $M$ is a $\pi$-group.

Now, it is obvious that $Z_{\pi} \subseteq M$. Let $r \in \pi\left(M / Z_{\pi}\right)$ and choose $R \in \operatorname{Syl}_{r}(M)$. Notice that $R \unlhd G$ and that $1 \neq[R, G] \leqslant[M, G]=N$. Therefore, $r \in \pi(N) \subseteq \pi\left(B_{0}\right)$ and thus 
$\pi\left(M / Z_{\pi}\right) \subseteq \pi\left(B_{0}\right)$ as wanted.

Finally, if $d^{G}=D$ is a generating class of $M$ and $R$ is a Sylow $r$-subgroup as above, then $\left|R: C_{R}(d)\right|$ divides $(|R|,|D|)=1$, so $R=C_{R}(d)$ and we get $R \leqslant \mathbf{Z}(M)$. Hence $M$ is Abelian.

LEMMA 4. Let $G$ be a $\pi$-separable group such that $\Gamma_{\pi}(G)$ has two connected components, $X_{1}$ and $X_{2}$, and assume that $X_{2}$ contains the maximal length classes. Then $|A|<|B|, A^{-1} A B=B$ and $\left|\left\langle A^{-1} A\right\rangle\right|$ divides $|B|$ for all $A \in X_{1}$ and all $B \in X_{2}$.

Proof: Let $B \in X_{2}, A \in X_{1}$ and choose $B_{0}$ any maximal length class. Since $(|A|,|B|)=1$ then $A B$ is again a class by Lemma $1(\mathrm{~b})$, satisfying that $|A B|$ divides $|A||B|$. As $A$ and $B$ belong to different components, we have two possibilities: either $|A B|=|A|$ or $|A B|=|B|$. If $|A B|=|A|$ then $B^{-1} B A=A$, and thus we deduce that $\left|\left\langle B^{-1} B\right\rangle\right|$ divides $|A|$ and $\left\langle B^{-1} B\right\rangle \subseteq\left\langle A A^{-1}\right\rangle$. But $\left|\left\langle A A^{-1}\right\rangle\right|$ also divides $\left|B_{0}\right|$ by Lemma 1 (c), so we conclude that $\left\langle\left\langle B^{-1} B\right\rangle\right|$ divides $\left(|A|,\left|B_{0}\right|\right)=1$, a contradiction. Consequently, $|A B|=|B|$, so in particular $|A|<|B|$. Moreover, $A^{-1} A B=B$ and then $\left|\left\langle A^{-1} A\right\rangle\right|$ divides $|B|$.

THEOREM 5. Suppose that $G$ is $\pi$-separable and that $\Gamma_{\pi}(G)$ has two connected components $X_{1}$ and $X_{2}$. Assume that $X_{2}$ contains the maximal length classes in $\operatorname{Con}\left(G_{\pi}\right)$ and let

$$
M=\left\langle D \in \operatorname{Con}\left(G_{\pi}\right) \mid D \in X_{1}\right\rangle
$$

Then

(a) $M$ coincides with the subgroup defined in Theorem 3. Accordingly, $M$ is an Abelian $\pi$-subgroup and $\pi\left(M / Z(G)_{\pi}\right) \subseteq \pi\left(X_{2}\right)$.

(b) there is no class in $X_{2}$ whose size is a $\pi^{\prime}$-number.

Proof: (a) Let $A \in X_{1}$ and let $B_{0}$ be a class of $\pi$-elements of maximal length. In Lemma 4 , we proved that $\left|\left\langle A A^{-1}\right\rangle\right|$ divides $|B|$ for every class $B \in X_{2}$. Thus, if $C \in \operatorname{Con}\left(G_{\pi}\right)$ with $\left(|C|,\left|B_{0}\right|\right)=1$, then either $C \in X_{1}$ or $|C|=1$. If $C=\{c\}$, then we need to show that $c \in M=\left\langle D \in \operatorname{Con}\left(G_{\pi}\right) \mid D \in X_{1}\right\rangle$. Observe that $c A=\{c a \mid a \in A\}$ is a conjugacy class of $\pi$-elements of $G$ with $|c A|=|A|$. Therefore, $c A \in X_{1}$, and $c a \in M$ for every $a \in A$. Since $a^{-1} \in M$, we conclude that $c \in M$.

(b) Fix a class $A \in X_{1}$. We know that $\left\langle A^{-1} A\right\rangle \subseteq M$, which is a $\pi$-group. For any class $B \in X_{2}$, we proved in Lemma 4 that $\left|\left\langle A^{-1} A\right\rangle\right|$ divides $|B|$, and thus, $|B|$ is not a $\pi^{\prime}$-number.

\section{Class lengths in $\pi$-Separable groups}

We state and prove now Theorem $\mathrm{C}$ of the Introduction.

THEOREM 6. Suppose that $G$ is $\pi$-separable and let $x \in G$ such that $\left|x^{G}\right|$ is a $\pi$-number. Then $\left[x^{G}, x^{G}\right] \subseteq \mathbf{O}_{\pi}(G)$. Consequently, $x \in \mathbf{O}_{\pi, \pi^{\prime}}(G)$. 
Proof: We argue by induction on $|G|$. As the hypotheses of the theorem are inherited by quotient groups we can assume that $\mathrm{O}_{\pi}(G)=1$ and we shall show that $\left[x^{G}, x^{G}\right]=1$.

Let $N=\left\langle x^{G}\right\rangle$ and suppose that $N<G$. Since $\mathrm{O}_{\pi}(N)=1$, it follows by induction that $\left[x^{N}, x^{N}\right]=1$. In particular, $\left\langle x^{N}\right\rangle$ is Abelian and subnormal in $G$. Thus $x \in\left\langle x^{N}\right\rangle$ $\subseteq F(G)$, which is a $\pi^{\prime}$-group. But since $\left|x^{G}\right|$ is a $\pi$-number, then $F(G) \subseteq C_{G}(x)$, and so $x$ is central in $F(G)$. Therefore, $\left\langle x^{G}\right\rangle$ is also central in $F(G)$, so we have $\left[x^{G}, x^{G}\right]=1$, as required.

Accordingly, we can assume that $N=G$. Now take $K$ a minimal normal subgroup of $G$, which must be a $\pi^{\prime}$-group. By assumption, we have $K \subseteq C_{G}(x)$ and, as $G=\left\langle x^{G}\right\rangle$, then $K \subseteq \mathbf{Z}(G)$. It follows that $\mathbf{O}_{\pi}(G / K)=\mathbf{O}_{\pi}(G) K / K=1$ and, by applying induction, we obtain $\left[(x K)^{G / K},(x K)^{G / K}\right]=1$, that is to say, $\left[x^{G}, x^{G}\right] \subseteq K \subseteq \mathrm{Z}(G)$. Then $G$ is nilpotent. Consequently, $G$ is a $\pi^{\prime}$-group, so $x$ is central in $G$ and the theorem is trivially true.

Now, $\left\langle x^{G}\right\rangle \mathrm{O}_{\pi}(G) / \mathrm{O}_{\pi}(G)$ is an Abelian normal subgroup of $G / \mathrm{O}_{\pi}(G)$. Hence

$$
\left\langle x^{G}\right\rangle \mathbf{O}_{\pi}(G) / \mathbf{O}_{\pi}(G) \subseteq F\left(G / \mathbf{O}_{\pi}(G)\right),
$$

which is necessarily a $\pi^{\prime}$-group. In particular, $x \in \mathbf{O}_{\pi, \pi^{\prime}}(G)$, as required.

REMARKS. Theorem 6 is a generalisation of the fact that if $G$ is a $\pi$-separable group, then every $x \in G_{\pi}$ with $\left|x^{G}\right|$ a $\pi$-number must lie in $\mathrm{O}_{\pi}(G)$ (see for instance, [9, Lemma 33.3]). On the other hand, Theorem 6 is simply not true when the $\pi$-separability hypothesis is eliminated. For instance, let $G$ be a nonabelian simple group and choose $x \neq 1$ to belong to the centre of a Sylow $p$-subgroup of $G$, for some prime $p$. Then $\left|x^{G}\right|$ is a $p^{\prime}$-number and $\mathbf{O}_{p^{\prime}}(G)=1$, while the property $\left[x^{G}, x^{G}\right]=1$ is clearly not satisfied.

We also need two lemmas. We shall denote by $l_{\pi}(G)$ the $\pi$-length of a group $G$.

LеммA 7. Let $G$ be a $\pi$-separable group. Then the conjugacy class length of any $\pi$-element in $G$ is a $\pi^{\prime}$-number if and only if $G$ has Abelian Hall $\pi$-subgroups. In this case, $l_{\pi}(G) \leqslant 1$.

ProOF: The first assertion can easily be proved by using induction on $|G|$ (it is exactly [3, Lemma 5]). The second assertion follows immediately by applying Theorem 6.

LEMMA 8: Let $G$ be a $\pi$-separable group. Then the conjugacy class length of every $\pi$-element of $G$ is a $\pi$-number if and only if $G=H \times K$, where $H$ and $K$ are a Hall $\pi$-subgroup and a $\pi$-complement of $G$, respectively.

Proof: Suppose first that $|C|$ is a $\pi$-number for any $C \in \operatorname{Con}\left(G_{\pi}\right)$ and take $H$ and $K$ a Hall $\pi$-subgroup and a $\pi$-complement of $G$ respectively. Then for each $x \in H$, there exists some $g \in G$ such that $K^{g} \subseteq C_{G}(x)$. Thus, $x \in C_{G}\left(K^{g}\right)$ and

$$
H \subseteq \bigcup_{g \in G} C_{G}\left(K^{g}\right)
$$


Since $G=H K$, we have

$$
H \subseteq \bigcup_{h \in H} C_{G}\left(K^{h}\right) \cap H \subseteq \bigcup_{h \in H} C_{H}(K)^{h} \subseteq H
$$

This yields $H=C_{H}(K)$ and then $G=H \times K$, as required. The converse direction of the lemma is trivial.

\section{Proofs of Theorem A and Corollary B}

Lemma 9. Let $G$ be a group. Suppose that $|C|$ is a prime power for any $C$ $\in \operatorname{Con}\left(G_{\pi}\right)$. Then $G$ is $\pi$-separable.

PROOF: If any $\pi$-element of $G$ is central, then $G$ trivially has a central Hall $\pi$ subgroup and the lemma is proved. Thus we can assume that there exists some $C$ $\in \operatorname{Con}\left(G_{\pi}\right)$ such that $1<|C|$ is a prime power. By Burnside's Lemma, $G$ is not simple, and then the result follows by induction on $|G|$, since the hypothesis is inherited by normal subgroups and quotients.

Suppose that all the conjugacy classes of elements in $G_{\pi}$ have prime power length. Then Theorem 2 implies that the primes appearing in this set of lengths can be at most two. Therefore, to show Theorem A of the Introduction, we only have to study two cases, depending on whether just one or two primes appear. Each one of these cases corresponds to Theorems 12 and 10 , respectively.

THEOREM 10. Let $G$ be a group and $\pi$ an arbitrary set of primes. Then the lengths of the conjugacy classes in $\operatorname{Con}\left(G_{\pi}\right)$ are powers of exactly two different primes $q$ and $r$ if and only if $q, r \in \pi$ and $G=H \times K$, where $K$ is a $\pi$-complement of $G$ and $H$ is a Hall $\pi$-subgroup which is quasi-Frobenius with Abelian kernel and complement of orders $q^{a}$ and $r^{b}$, with $a, b>0$. In particular, the set of conjugacy class lengths in $\operatorname{Con}\left(G_{\pi}\right)$. is exactly $\left\{1, q^{a}, r^{b}\right\}$.

Proof: First, we note that $G$ is $\pi$-separable by Lemma 9. Suppose that the lengths of the classes in $\operatorname{Con}\left(G_{\pi}\right)$ are powers of exactly two primes, $q$ and $r$, and suppose without loss that $q^{a}$, with $a>0$, is the maximal size. Then $\Gamma_{\pi}(G)$ has two connected components and Theorem 5(b) asserts that $q \in \pi$. Now, we use the subgroup $M$ defined in the statement of Theorem 5. By definition, we have $M=\left\langle D \in \operatorname{Con}\left(G_{\pi}\right)|| D\right|$ is an $r$-power $\rangle$, which further is an Abelian $\pi$-group by Theorem 5(a). We claim that $G$ has a normal Hall $\pi$-subgroup. Let us consider $G / M$, which satisfies that all conjugacy classes of $\pi$-elements have $q$-power length. Then, by Lemma 8 , we can factor $G / M=H / M \times K M / M$, where $H$ and $K$ are a Hall $\pi$-subgroup and a $\pi$-complement of $G$, respectively. Accordingly, $G$ has a normal Hall $\pi$-subgroup, as claimed. Also, we shall use later the fact that $K M \unlhd G$, for any $\pi$-complement $K$ of $G$.

Notice that the conjugacy class lengths of $H$ are also $r$-powers or $q$-powers, since they divide the conjugacy class sizes of $G$. If we prove that $H$ has conjugacy classes 
whose size are $q$-numbers and $r$-numbers, we shall obtain, via [5, Theorem A], that $H$ is quasi-Frobenius with Abelian kernel and complement. Suppose first that every class in $H$ has $r$-power size and assume that $M<H$. Take $x \in H-M$ and observe that $\left|x^{G}\right|$ must be a $q$-number by definition of $M$, so $\left|x^{H}\right|=1$. This forces $H=M \mathrm{Z}(H)$, and thus $H$ is Abelian (observe that if $M=H$ then $H$ is also Abelian). It follows that the length of every $C \in \operatorname{Con}\left(G_{\pi}\right)$ is a $\pi^{\prime}$-number, contradicting the fact that $q \in \pi$.

Suppose now that all the conjugacy classes in $H$ have $q$-power size and take $x \in H$ such that $\left|x^{G}\right|$ is an $r$-power. Then $\left|x^{H}\right|=1$, that is, $x \in \mathbf{Z}(H)$, and therefore, $M \subseteq \mathbf{Z}(H)$. If $H=M$, we get a contradiction as above. Thus we can take $x \in H-M$. By definition of $M, 1 \neq\left|x^{G}\right|$ is a $q$-number, so in particular, it is a $\pi$-number. Then $K^{g} \subseteq C_{G}(x)$ for some $g \in G$. But on the other hand, we have proved that $M \subseteq C_{G}(x)$, and then $K M=K^{g} M \subseteq C_{G}(x)$ for every $x \in H-M$. We conclude that $K \subseteq C_{G}(H)$ and this means that all conjugacy classes in $G_{\pi}$ have $q$-power length, a contradiction.

Thus, we have shown that $H$ has conjugacy classes of $r$-power size and classes of $q$-power size. Therefore $q, r \in \pi$, and by Lemma 8, we obtain $G=H \times K$. Moreover, from the fact that $H$ is quasi-Frobenius with Abelian kernel and complement one can easily check that the conjugacy class sizes of $H$ are exactly $\left\{1, q^{a}, r^{b}\right\}$ for some positive integers $a, b$, where $q^{a}$ and $r^{b}$ are exactly the orders of the kernel and a complement of $H$, respectively.

The converse direction of the theorem is trivial.

The following is Corollary $B$ of the Introduction.

COROLLARY 11. Let $G$ be a group and $p$ a fixed prime such that all the conjugacy classes of p-elements have prime power size. Then there exists only one prime $q$, equal to or distinct from $p$, such that all classes of $p$-elements have $q$-power size.

Proof: Take $\pi=\{p\}$ in Theorem 10. By Theorem 2 there exist at most two primes dividing the conjugacy class sizes in $G_{\pi}$. If there are exactly two, then they must belong to $\pi$ by Theorem 10 , and this is not possible.

Now we study the remaining case, that is, when all classes in $G_{\pi}$ have $q$-power size for a fixed prime $q$.

THEOREM 12. Suppose that for any $C \in \operatorname{Con}\left(G_{\pi}\right),|C|$ is a power of a fixed prime q. Then

(a) $q \notin \pi$ if and only if $G$ has an Abelian Hall $\pi$-subgroup $H$. In this case $H \mathrm{O}_{q}(G) \unlhd G$.

(b) $q \in \pi$ if and only if $G=H \times K$ where $K$ is a $\pi$-complement and $\dot{H}$ is a nilpotent Hall $\pi$-subgroup having all Abelian Sylow subgroups except for the prime $q$.

Furthermore, in both cases $l_{\pi}(G) \leqslant 1$ and $G / \mathrm{O}_{\pi^{\prime}}(G)$ is solvable.

Proof: (a) The direct sense is immediate by Lemmas 7 and 9 , and the converse 
direction is trivial. In this case, it remains to be proved that if $H$ is a Hall $\pi$-subgroup of $G$ then $H \mathrm{O}_{q}(G) \unlhd G$ and that $G / \mathrm{O}_{\pi^{\prime}}(G)$ is solvable.

We first notice that $l_{\pi}(G) \leqslant 1$ by applying Lemma 7 . To prove that $H \mathbf{O}_{q}(G)$ is normal in $G$, we first see, arguing by induction on $|G|$, that we may assume that $\mathbf{O}_{\pi}(G)$ $=1$. If $1 \neq M:=\mathbf{O}_{\pi}(G)$ then, as the hypotheses are inherited by factor groups, by induction we have $\mathrm{O}_{q}(G / M) H / M \unlhd G / M$. On the other hand, we can write $\mathbf{O}_{q}(G / M)$ $=Q_{0} M / M$ for some $q$-subgroup $Q_{0}$ of $G$, and thus $H Q_{0} \unlhd G$. Moreover, $\mathbf{O}_{q}\left(H Q_{0}\right)$ $=\mathrm{O}_{q}(G)$ since $\mathrm{O}_{q}(G) \subseteq Q_{0} M \subseteq H Q_{0}$. Now we distinguish two possibilities. If $H Q_{0}<G$, by induction it follows that $H \mathbf{O}_{q}(G) \unlhd H Q_{0}$. This implies that $H \mathrm{O}_{q}(G) \unlhd G$, because we observe that $H \mathrm{O}_{q}(G) / \mathbf{O}_{q}(G)=\mathbf{O}_{\pi}\left(H Q_{0} / \mathbf{O}_{q}(G)\right)$. If $H Q_{0}=G$ then $\mathbf{O}_{\pi^{\prime}}(G)=\mathbf{O}_{q}(G)$ and since $l_{\pi}(G) \leqslant 1$, we also conclude that $H \mathbf{O}_{q}(G) \unlhd G$, as required.

We prove now that we can also assume that $\mathrm{O}_{\pi^{\prime}, \pi}(G)=G$, that is, $\mathrm{O}_{\pi^{\prime}}(G) H=G$. Suppose that $N=$ : $\mathbf{O}_{\pi^{\prime}}(G) H<G$. As $l_{\pi}(G) \leqslant 1$, then $N \triangleleft G$. Since the hypotheses are inherited by normal subgroups, by applying induction we get $H \mathrm{O}_{q}(N) \unlhd N$, and thus $H \mathrm{O}_{q}(N) \unlhd G$. But notice that $\mathrm{O}_{q}(N)=\mathrm{O}_{q}(G)$, so we conclude that $H \mathrm{O}_{q}(G)$ is normal in $G$ and the proof finishes.

Now, for any $x \in H$ and any prime $r \in \pi^{\prime}, r \neq q$, by assumption there exists some $R \in \operatorname{Syl}_{r}(G)$ such that $R \subseteq C_{G}(x)$, that is, $x \in C_{G}(R)$. Hence

$$
H \subseteq \bigcup_{g \in G} C_{G}(R)^{g}
$$

Then

$$
G=H \mathbf{O}_{\pi^{\prime}}(G) \subseteq \bigcup_{g \in G} C_{G}\left(R^{g}\right) \mathbf{O}_{\pi^{\prime}}(G) \subseteq G,
$$

so we have $G=C_{G}(R) \mathbf{O}_{\pi^{\prime}}(G)$ for some $R \in \operatorname{Syl}_{r}(G)$. In particular, $\left|G: C_{G}(R)\right|$ is a $\pi$-number and, replacing by some $G$-conjugated of $R$, we may assume that $H \subseteq C_{G}(R)$, or equivalently, $R \subseteq C_{G}(H)$. This has been proved for every prime $r \in \pi^{\prime}-\{q\}$ and since $H$ is Abelian, it follows that $\left|G: C_{G}(H)\right|$ is a $q$-number. Then $G=C_{G}(H) Q$ for some $Q \in \operatorname{Syl}_{q}(G)$. Furthermore, we can choose $Q$ to be normalised by $H$, because, by the Frattini argument, $G=N_{G}(Q) \mathbf{O}_{\pi^{\prime}}(G)$, so in particular, $N_{G}(Q)$ has $\pi^{\prime}$-index in $G$, and consequently, there exists some Hall $\pi$-subgroup of $G$ normalising $Q$.

Let us consider $[H, G]=\left[H, C_{G}(H) Q\right]=[H, Q] \leqslant Q$. Then $[H, G]$ is a normal $q$-subgroup of $G$, which cannot be trivial because this contradicts the assumption $O_{\pi}(G)$ $=1$. This forces $\mathrm{O}_{q}(G) \neq 1$ and then we can apply the inductive hypothesis to $G / \mathrm{O}_{q}(G)$ to conclude that $H \mathrm{O}_{q}(G) \unlhd G$ and the proof is finished.

Finally, the solvability of $G / \mathrm{O}_{\pi^{\prime}}(G)$ is a consequence of $[7$, Theorem $\mathrm{A}(\mathrm{d})]$, which asserts that, under our hypotheses, $G / \mathrm{O}_{r^{\prime}}(G)$ is solvable for any prime $r \in \pi$. In fact, it is enough to notice that $\mathrm{O}_{\pi^{\prime}}(G)=\cap_{r \in \pi} \mathrm{O}_{r^{\prime}}(G)$ and that $G / \mathrm{O}_{\pi^{\prime}}(G)$ can be immerged into $\prod_{r \in \pi} G / \mathbf{O}_{r^{\prime}}(G)$, which is solvable too. 
(b) Suppose that $q \in \pi$. Then $G=H \times K$ by Lemma 8 . Notice that the conjugacy class sizes of $H$ are also $q$-powers, and this implies (see for instance [8, Proposition 4]) that $H=Q \times L$ with $Q$ a Sylow $q$-subgroup of $H$ and $L$ Abelian, as wanted. Conversely, if $G$ is as described in the statement, it clearly follows that the conjugacy classes of $G_{\pi}$ have $q$-power size and that $q \in \pi$. Finally, the fact that $l_{\pi}(G) \leqslant 1$ and the solvability of $G / \mathbf{O}_{\pi^{\prime}}(G)$ are trivial in this case.

REMARK. Let us assume the hypotheses of Theorem 12 with $\pi=\{p\}$. Then we have provided a more elementary proof of the fact that $\mathrm{O}_{q}(G) P \unlhd G$, with $P \in \operatorname{Syl}_{p}(G)$, which is part of $[\mathbf{7}$, Theorem $A(d)]$.

\section{REFERENCES}

[1] R. Baer, 'Group elements of prime power index', Trans. Amer. Math. Soc. 75 (1953), $20-47$.

[2] A. Beltrán and M.J. Felipe, 'On the diameter of a p-regular conjugacy class graph of finite groups', Comm. Algebra 30 (2002), 5861-5873.

[3] A. Beltrán and M.J. Felipe, 'Finite groups with a disconnected $p$-regular conjugacy class graph', Comm. Algebra (to appear).

[4] A. Beltrán and M.J. Felipe, 'Certain relations between p-regular class sizes and the $p$-structure of $p$-solvable groups', J. Austral. Math. Soc. (to appear).

[5] E.A. Bertram, M. Herzog and A. Mann, 'On a graph related to conjugacy classes of groups', Bull. Lond. Math. Soc. 22 (1990), 569-575.

[6] M. Bianchi, D. Chillag, A.G. Mauri, M. Herzog and C.M. Scoppola, 'Applications of a graph related to conjugacy classes in finite groups', Arch. Math. 58 (1992), 126-132.

[7] A.R. Camina and R.D. Camina, 'Implications of conjugacy class size', J. Group Theory 1 (1998), 257-269.

[8] D. Chillag and M. Herzog, 'On the lengths of the conjugacy classes of finite groups', $J$. Algebra 131 (1990), 110-125.

[9] B. Huppert, Character theory of finite groups, De Gruyter Expositions in Mathematics 25 (Walter de Gruyter \& Co, Berlin, 1998).

[10] S.L. Kazarin, 'Burnside's $p^{\alpha}$-lemma', (in Russian), Mat. Zametki 48 (1990), 45-48, 158. English translation, Math Notes 48 (1990), 749-751.

[11] Z. Lu and J. Zhang, 'On the diameter of a graph related to p-regular conjugacy classes of finite groups', J. Algebra 231 (2000), 705-712.

Departamento de Matemáticas, Universidad Jaume I, 12071 Castellón Spain e-mail: abeltran@mat.uji.es

\author{
Departamento de Matemática Aplicada, \\ Universidad Politécnica de Valencia, \\ 46022 Valencia \\ Spain \\ e-mail: mfelipe@mat.upv.es
}

